\title{
ON A TERMINATING SHOCK PROCESS WITH INDEPENDENT WEAR INCREMENTS
}

\author{
JI HWAN CHA, ${ }^{*}$ Ewha Womans University \\ MAXIM FINKELSTEIN, ${ }^{* *}$ University of the Free State and \\ Max Planck Institute for Demographic Research
}

\begin{abstract}
In extreme shock models, only the impact of the current, possibly fatal shock is usually taken into account, whereas in cumulative shock models, the impact of the preceding shocks is accumulated as well. In this paper we combine an extreme shock model with a specific cumulative shock model. It is shown that the proposed setting can also be interpreted as a generalization of the well-known Brown-Proschan model that describes repair actions for repairable systems. For a system subject to a specific process of shocks, we derive the survival probability and the corresponding failure rate function. Some meaningful interpretations and examples are discussed.
\end{abstract}

Keywords: Brown-Proschan model; extreme shock model; cumulative shock model; virtual age; wear

2000 Mathematics Subject Classification: Primary 60K10

Secondary 62P30

\section{Introduction}

Consider first a repairable system with the underlying absolutely continuous cumulative distribution function (CDF) $F(t)$ and the corresponding failure rate $\lambda(t)$. Assume that each repair is perfect with probability $p(t)$ and is minimal with probability $q(t)=1-p(t)$. Denote by $F_{p}(t)$ the CDF of the time between two consecutive perfect repairs. Also, let

$$
\int_{0}^{\infty} p(u) \lambda(u) \mathrm{d} u=\infty,
$$

which ensures that $F_{p}(t)$ is a proper distribution $\left(F_{p}(\infty)=1\right)$. Then

$$
F_{p}(t)=1-\exp \left\{-\int_{0}^{t} p(u) \lambda(u) \mathrm{d} u\right\} .
$$

For the case $p(t) \equiv p$, this model was considered in Brown and Proschan (1983), and, therefore, in the literature it is usually called the Brown-Proschan model.

Now consider a system subject to a nonhomogeneous Poisson process of shocks with rate $v(t)$. Let it be 'absolutely reliable' in the absence of shocks. Assume that each shock (regardless of its number) results in the system's failure (and, therefore, in the termination of

Received 3 November 2008; revision received 9 March 2009.

* Postal address: Department of Statistics, Ewha Womans University, Seoul, 120-750, Korea.

Email address: jhcha@ewha.ac.kr

** Postal address: Department of Mathematical Statistics, University of the Free State, PO Box 339, Bloemfontein 9300, Republic of South Africa. Email address: finkelm.sci@ufs.ac.za 
the corresponding Poisson shock process) with probability $p(t)$ and is harmless to the system with probability $q(t)=1-p(t)$. Denote the corresponding time to failure of the system by $T_{S}$. It is obvious that the described setting is a mathematically equivalent reformulation of the Brown-Proschan model and, therefore, the corresponding survival probability in this case can be defined similar to (1), i.e.

$$
\mathrm{P}\left(T_{S}>t\right) \equiv \bar{F}_{S}(t)=\exp \left\{-\int_{0}^{t} p(u) v(u) \mathrm{d} u\right\} .
$$

The formal proof of (1) can be found in Beichelt and Fischer (1980) and Block et al. (1985). A 'nontechnical proof', based on the notion of the conditional intensity function (see Cox and Isham (1980)) is given in, e.g. Nachlas (2005) and Finkelstein (2008). Note that the described shocks related setting is often referred to in the literature as an extreme shock model, as only the impact of the current, possibly fatal shock is taken into account, whereas in cumulative shock models (see later) the impact of all shocks is accumulated (see Sumita and Shanthikumar (1985) and Gut and Husler (2005), among others). The failure in the latter model occurs when the corresponding accumulated value reaches the predetermined boundary.

It is clear that the extreme shock model can be easily modified to the case when a system can also fail from causes other than shocks. Denote the corresponding CDF in the absence of shocks by $F(t)$ and assume that the corresponding process of failure is independent of our shock process. It follows from the competing risks consideration that

$$
\mathrm{P}\left(T_{S}>t\right)=\bar{F}(t) \exp \left\{-\int_{0}^{t} p(u) v(u) \mathrm{d} u\right\} .
$$

A crucial assumption for obtaining (2) and (3) is the assumption that, with probability $q(t)=1-p(t)$, a shock does not result in any changes in a system, which is an analogue of minimal repair in the Brown-Proschan model. However, it is not often the case in practice as shocks usually increase deterioration, wear, etc, which is analogous to 'worse than minimal repair'. The effect of different shocks is also usually accumulated in some way. In this paper we will combine the extreme shock model with a specific accumulated shocks model to be defined in the next section. Note that Gut and Husler (2005) considered asymptotic properties (as $t \rightarrow \infty$ ) for the specific combination of the accumulated shocks model with a one-shock process that can decrease the boundary for the accumulated wear.

Taking the settings discussed in this section into account, the following model can also be interpreted as a generalization of the Brown-Proschan model.

\section{The model}

Denote, for convenience of notation, a system's lifetime in a baseline environment (without shocks) by $R$. Thus, $\mathrm{P}(R \leq t)=F(t)$. Here we interpret $R$ as some initial, random resource, which is 'consumed' by a system in the process of its operation with rate 1 . Therefore, the age of our system in this case is equal to a calendar time $t$, and a failure occurs when this age reaches $R$. It is clear that as the remaining resource decreases with time, our system can be considered to be ageing (deteriorating).

Let $N(t), t \geq 0$, denote an orderly point process of shocks with arrival times $T_{i}, i=$ $1,2, \ldots$, and let the CDF of a system's lifetime $T_{S}$ in the presence of shocks, as in Section 1 , be $F_{S}(t)$. Assume that the $i$ th shock, as in the Brown-Proschan model, causes immediate system failure with probability $p(t)$, but in contrast to this model, with probability $q(t)$, it now 
increases the age of a system by a random increment $W_{i} \geq 0$. In terms of repair actions, this repair is worse than minimal. In accordance with this setting, the random age of a system at time $t$ (which corresponds to the 'virtual age' of Finkelstein (2007), (2008)) is

$$
T_{v}=t+\sum_{i=0}^{N(t)} W_{i}
$$

where, formally, $W_{0}=0$ corresponds to the case in which $N(t)=0$ when there are no shocks in $[0, t]$. Failure occurs when this random variable reaches the boundary $R$. Therefore,

$$
\begin{aligned}
\mathrm{P}\left(T_{S}\right. & \left.>t \mid N(s), 0 \leq s \leq t ; W_{1}, W_{2}, \ldots, W_{N(t)} ; R\right) \\
& =\prod_{i=0}^{N(t)} q\left(T_{i}\right) \mathbf{1}\left(T_{v} \leq R\right) \\
& =\prod_{i=0}^{N(t)} q\left(T_{i}\right) \mathbf{1}\left(\sum_{i=0}^{N(t)} W_{i} \leq R-t\right)
\end{aligned}
$$

where $q\left(T_{0}\right)=1$ also corresponds to the case in which $N(t)=0$ and $\mathbf{1}(x)$ is the corresponding indicator. This probability should be understood conditionally on the corresponding realizations of $N(t), W_{i}, i=1,2, \ldots, N(t)$, and $R$.

Relationship (4) is very general and it is impossible to 'integrate out' explicitly $N(t), W_{i}, i=$ $1,2, \ldots, N(t)$, and $R$ without substantially simplifying the assumptions. In the next section we will consider two important specific cases.

The described model can be equivalently formulated in the following way. Let $F(t)$ describe the deterioration of the wearing item in the baseline environment. Failure occurs when this wear, which in the standardized form is equal to $t$, reaches the resource $R$. Denote the random wear in a more severe environment by $W_{t}, t \geq 0$. Specifically, for our shock model, $W_{t}=t+\sum_{i=0}^{N(t)} W_{i}$, where the $W_{i}, i=1,2, \ldots, N(t)$, are the random increments of wear due to shocks and $W_{0} \equiv 0$ (see Finkelstein (1999)). For convenience, in what follows we will use this wear-based interpretation.

\section{Special cases}

\subsection{Exponentially distributed boundary}

In addition to the assumptions of Section 2, we adopt the following assumptions.

Assumption 1. $N(t), t \geq 0$, is the nonhomogeneous Poisson process with rate $v(t)$.

Assumption 2. The $W_{i}, i=1,2, \ldots$, are independent and identically distributed (i.i.d.) random variables characterized by the moment generating function $M_{W}(t)$ and the $C D F G(t)$.

Assumption 3. $N(t), t \geq 0 ; W_{i}, i=1,2, \ldots$; and $R$ are independent of each other.

Assumption 4. $R$ is exponentially distributed with the failure rate $\lambda$, i.e. $\bar{F}(t)=\exp \{-\lambda t\}$.

The following result gives the survival function and the failure rate function of $T_{S}$.

Theorem 1. Let $m(t) \equiv \mathrm{E}[N(t)]=\int_{0}^{t} v(x) \mathrm{d} x$. Suppose that Assumptions 1-4 hold and that the inverse function $m^{-1}(t)$ exists. Then the survival function of $T_{S}$ and the corresponding 
failure rate $\lambda_{S}(t)$ are given by

$$
\mathrm{P}\left(T_{S}>t\right)=\exp \left\{-\lambda t-\int_{0}^{t} v(x) \mathrm{d} x+M_{W}(-\lambda) \int_{0}^{t} q(x) v(x) \mathrm{d} x\right\}, \quad t \geq 0,
$$

and

$$
\lambda_{S}(t)=\lambda+\left(1-M_{W}(-\lambda) q(t)\right) v(t)
$$

respectively.

Proof. Given the assumptions, we can directly 'integrate out' the variable $R$ and define the corresponding probability in the following way:

$$
\begin{aligned}
\mathrm{P}\left(T_{S}\right. & \left.>t \mid N(s), 0 \leq s \leq t, W_{1}, W_{2}, \ldots, W_{N(t)}\right) \\
& =\left(\prod_{i=0}^{N(t)} q\left(T_{i}\right)\right) \exp \left\{-\int_{0}^{t+\sum_{i=0}^{N(t)} W_{i}} \lambda \mathrm{d} u\right\} \\
& =\exp \left\{-\lambda t-\lambda \sum_{i=1}^{N(t)} W_{i}+\sum_{i=1}^{N(t)} \ln q\left(T_{i}\right)\right\} .
\end{aligned}
$$

Thus, we have

$$
\begin{aligned}
\mathrm{P}\left(T_{S}\right. & >t \mid N(s), 0 \leq s \leq t) \\
& =\exp \{-\lambda t\} \exp \left\{\sum_{i=1}^{N(t)} \ln q\left(T_{i}\right)\right\} \mathrm{E}\left[\exp \left\{-\sum_{i=1}^{N(t)} \lambda W_{i}\right\}\right] \\
& =\exp \{-\lambda t\} \exp \left\{\sum_{i=1}^{N(t)} \ln q\left(T_{i}\right)\right\}\left(M_{W}(-\lambda)\right)^{N(t)} \\
& =\exp \{-\lambda t\} \exp \left\{\sum_{i=1}^{N(t)}\left[\ln q\left(T_{i}\right)+\ln \left(M_{W}(-\lambda)\right)\right]\right\} .
\end{aligned}
$$

Define $N^{*}(t) \equiv N\left(m^{-1}(t)\right), t \geq 0$, and $T_{j}^{*} \equiv m\left(T_{j}\right), j \geq 1$. It is known that $\left\{N^{*}(t), t \geq 0\right\}$ is a stationary Poisson process with intensity 1 (see, e.g. Çinlar (1975)), and $T_{j}^{*}, j \geq 1$, are the times of occurrence of shocks in the new time scale. Let $s=m(t)$. Then

$$
\begin{aligned}
\mathrm{E}[\exp & \left.\left\{\sum_{i=1}^{N(t)}\left[\ln q\left(T_{i}\right)+\ln \left(M_{W}(-\lambda)\right)\right]\right\}\right] \\
& =\mathrm{E}\left[\exp \left\{\sum_{i=1}^{N^{*}(s)}\left[\ln q\left(m^{-1}\left(T_{i}^{*}\right)\right)+\ln \left(M_{W}(-\lambda)\right)\right]\right\}\right] \\
& =\mathrm{E}\left[\mathrm{E}\left[\exp \left\{\sum_{i=1}^{N^{*}(s)}\left[\ln q\left(m^{-1}\left(T_{i}^{*}\right)\right)+\ln \left(M_{W}(-\lambda)\right)\right]\right\} \mid N^{*}(s)\right]\right] .
\end{aligned}
$$

The joint distribution of $\left(T_{1}^{*}, T_{2}^{*}, \ldots, T_{n}^{*}\right)$ given $N^{*}(s)=n$ is the same as the joint distribution of $\left(V_{(1)}, V_{(2)}, \ldots, V_{(n)}\right)$, where $V_{(1)} \leq V_{(2)} \leq \cdots \leq V_{(n)}$ are the order statistics of i.i.d. random 
variables $V_{1}, V_{2}, \ldots, V_{n}$ which are uniformly distributed in the interval $[0, s]=[0, m(t)]$. In (7), the conditional expectation is given by

$$
\begin{aligned}
\mathrm{E}[\exp & \left.\left\{\sum_{i=1}^{N^{*}(s)}\left(\ln q\left(m^{-1}\left(T_{i}^{*}\right)\right)+\ln \left(M_{W}(-\lambda)\right)\right)\right\} \mid N^{*}(s)=n\right] \\
& =\mathrm{E}\left[\exp \left\{\sum_{i=1}^{n}\left(\ln q\left(m^{-1}\left(T_{i}^{*}\right)\right)+\ln \left(M_{W}(-\lambda)\right)\right)\right\} \mid N^{*}(s)=n\right] \\
& =\mathrm{E}\left[\exp \left\{\sum_{i=1}^{n}\left(\ln q\left(m^{-1}\left(V_{(i)}\right)\right)+\ln \left(M_{W}(-\lambda)\right)\right)\right\}\right] \\
& =\mathrm{E}\left[\exp \left\{\sum_{i=1}^{n}\left(\ln q\left(m^{-1}\left(V_{i}\right)\right)+\ln \left(M_{W}(-\lambda)\right)\right)\right\}\right] \\
& =\left(\mathrm{E}\left[\exp \left\{\ln q\left(m^{-1}\left(V_{1}\right)\right)+\ln \left(M_{W}(-\lambda)\right)\right\}\right]\right)^{n} \\
& =\left(\mathrm{E}\left[\exp \left\{\ln q\left(m^{-1}(s U)\right)+\ln \left(M_{W}(-\lambda)\right)\right\}\right]\right)^{n},
\end{aligned}
$$

where $U \equiv V_{1} / s=V_{1} / m(t)$ is a random variable uniformly distributed in the unit interval $[0,1]$. Therefore,

$$
\begin{aligned}
& \mathrm{E}\left[\exp \left\{\ln q\left(m^{-1}(s U)\right)+\ln \left(M_{W}(-\lambda)\right)\right\}\right] \\
& \quad=\int_{0}^{1} \exp \left\{\ln q\left(m^{-1}(s u)\right)+\ln \left(M_{W}(-\lambda)\right)\right\} \mathrm{d} u \\
& \quad=\int_{0}^{1} \exp \left\{\ln q\left(m^{-1}(m(t) u)\right)+\ln \left(M_{W}(-\lambda)\right)\right\} \mathrm{d} u \\
& \quad=\frac{M_{W}(-\lambda)}{m(t)} \int_{0}^{t} q(x) v(x) \mathrm{d} x
\end{aligned}
$$

Hence,

$$
\begin{gathered}
\mathrm{E}\left[\exp \left\{\sum_{i=1}^{N^{*}(s)}\left(\ln q\left(m^{-1}\left(T_{i}^{*}\right)\right)+\ln \left(M_{W}(-\lambda)\right)\right)\right\} \mid N^{*}(s)=n\right] \\
=\left(\frac{M_{W}(-\lambda)}{m(t)} \int_{0}^{t} q(x) v(x) \mathrm{d} x\right)^{n} .
\end{gathered}
$$

From (6), (7), and (8),

$$
\begin{aligned}
\mathrm{P}\left(T_{S}>t\right) & =\exp \{-\lambda t\} \sum_{n=0}^{\infty}\left(\frac{M_{W}(-\lambda)}{m(t)} \int_{0}^{t} q(x) v(x) \mathrm{d} x\right)^{n} \frac{s^{n}}{n !} \mathrm{e}^{-s} \\
& =\exp \{-\lambda t\} \mathrm{e}^{-s} \exp \left\{M_{W}(-\lambda) \frac{s}{m(t)} \int_{0}^{t} q(x) v(x) \mathrm{d} x\right\} \\
& =\exp \left\{-\lambda t-\int_{0}^{t} v(x) \mathrm{d} x+M_{W}(-\lambda) \int_{0}^{t} q(x) v(x) \mathrm{d} x\right\} .
\end{aligned}
$$

Therefore, the failure rate function of the system, $\lambda_{S}(t)$, is given by

$$
\lambda_{S}(t)=\lambda+\left(1-M_{W}(-\lambda) q(t)\right) v(t) .
$$

This completes the proof. 
The following corollary defines the failure rate function of $T_{S}$ when the $W_{i}$ s are distributed exponentially with mean $\mu$.

Corollary 1. If the $W_{i}$ s are distributed exponentially with mean $\mu$ then the failure rate function $\lambda_{S}(t)$ is given by

$$
\lambda_{S}(t)=\lambda+\left(1-\frac{q(t)}{\lambda \mu+1}\right) \nu(t) .
$$

Remark 1. This approach can be generalized to some extent to arbitrary $\lambda(t)$ and nonidentical $W_{i}$. For instance, the diverging geometric process for increments $W_{i}$, i.e. $W_{i+1}=a W_{i}, a>$ 1 , can be considered, but it is not possible to present these results in a simple and self-explanatory form similar to (5).

The following remark deals with a qualitative analysis of (5) and (9).

Remark 2. (i) Equation (5) suggests that the failure rate $\lambda_{S}(t)$ can be interpreted as a failure rate of a series system with dependent (via $R$ ) components.

(ii) As $M_{W}(0)=1$ for $\lambda=0, \lambda_{S}(t)$ for this case reduces to the failure rate of the BrownProschan model, (2).

(iii) When $\mu \rightarrow \infty$, from (9), we obtain $\lambda_{S}(t) \rightarrow \lambda+v(t)$, which means that a failure occurs either in accordance with the baseline $F(t)$ or as a result of the first shock (competing risks). Note that, in accordance with the properties of Poisson processes, the rate $v(t)$ is equal to the failure rate, which corresponds to the time to the first shock. Therefore, the two 'components' of the described series system are asymptotically independent as $\mu \rightarrow \infty$.

(iv) When $\mu=0$, which means that $W_{i}=0, i \geq 1$, (9) becomes $\lambda_{S}(t)=\lambda+p(t) v(t)$. Therefore, this specific case describes the series system with two independent components. The first component has the failure rate $\lambda$ and the second component has the failure rate $p(t) v(t)$, which corresponds to the failure rate in the Brown-Proschan model (see (3)).

(v) It also follows from (5) that when $p(t)=p$ and $v(t)=v$, the failure rate $\lambda_{S}(t)$ is also constant, which is a remarkable fact.

(vi) Let $q(t)=1$ (no killing shocks), and let $W_{i}$ be deterministic and equal to $\mu$. Then $M_{W}(-\lambda)=\exp \{-\mu \lambda\}$, and (5) becomes

$$
\lambda_{S}(t)=\lambda+(1-\exp \{-\mu \lambda\}) v(t)
$$

which can also be obtained easily by direct derivation.

Remark 3. Equation (9) can be generalized to the case when the $W_{i}$ s follow the gamma distribution with parameters $\alpha$ and $\mu$, i.e.

$$
\lambda_{S}(t)=\lambda+\left(1-\frac{q(t)}{(\lambda \mu+1)^{\alpha}}\right) v(t) .
$$

\subsection{Deterministic boundary}

Let $R=b$ be the deterministic boundary. Let other assumptions of Subsection 3.1 (Assumptions 1-3) hold. We consider the case in which $t<b$, which means that a failure cannot occur without shocks. The following result gives the survival function of $T_{S}$. 
Theorem 2. Suppose that Assumptions 1-3 hold and that the inverse function $m^{-1}(t)$ exists. Furthermore, let the $W_{i}$ s be i.i.d. exponential with mean $1 / \eta$. Then the survival function of $T_{S}$ is given by

$$
\begin{aligned}
\mathrm{P}\left(T_{S}>t\right)=\sum_{n=0}^{\infty} & \left(\sum_{j=n}^{\infty} \frac{(\eta(b-t))^{j}}{j !} \exp \{-\eta(b-t)\}\right)\left(\frac{1}{m(t)} \int_{0}^{t} q(x) v(x) \mathrm{d} x\right)^{n} \\
& \times \frac{m(t)^{n}}{n !} \exp \{-m(t)\}, \quad 0 \leq t<b .
\end{aligned}
$$

Proof. In this case, as in Subsection 3.1,

$$
\begin{aligned}
\mathrm{P}\left(T_{S}\right. & \left.>t \mid N(s), 0 \leq s \leq t, W_{1}, W_{2}, \ldots, W_{N(t)}\right) \\
& =\left(\prod_{i=1}^{N(t)} q\left(T_{i}\right)\right) \mathbf{1}\left(t+\sum_{i=1}^{N(t)} W_{i} \leq b\right) .
\end{aligned}
$$

Thus, we have

$$
\begin{aligned}
\mathrm{P}\left(T_{S}>t \mid N(s), 0 \leq s \leq t\right) & =\left(\prod_{i=1}^{N(t)} q\left(T_{i}\right)\right) \mathrm{P}\left(\sum_{i=1}^{N(t)} W_{i} \leq b-t\right) \\
& =\left(\prod_{i=1}^{N(t)} q\left(T_{i}\right)\right) G^{(N(t))}(b-t),
\end{aligned}
$$

where $G^{(n)}(t)$ is the $n$-fold convolution of $G(t)$ with itself.

As a special case, when the $W_{i}$ s are i.i.d. exponential with mean $1 / \eta$,

$$
\mathrm{P}\left(T_{S}>t \mid N(s), 0 \leq s \leq t\right)=\left(\prod_{i=1}^{N(t)} q\left(T_{i}\right)\right) \Psi(N(t))
$$

where

$$
\Psi(N(t)) \equiv \sum_{j=N(t)}^{\infty} \frac{(\eta(b-t))^{j}}{j !} \exp \{-\eta(b-t)\}
$$

and

$$
\begin{aligned}
\mathrm{P}\left(T_{S}>t\right) & =\mathrm{E}\left[\left(\prod_{i=1}^{N(t)} q\left(T_{i}\right)\right) \Psi(N(t))\right] \\
& =\mathrm{E}\left[\mathrm{E}\left[\left(\prod_{i=1}^{N(t)} q\left(T_{i}\right)\right) \Psi(N(t)) \mid N(t)\right]\right]
\end{aligned}
$$

where

$$
\mathrm{E}\left[\left(\prod_{i=1}^{N(t)} q\left(T_{i}\right)\right) \Psi(N(t)) \mid N(t)=n\right]=\Psi(n) \mathrm{E}\left[\left(\prod_{i=1}^{N(t)} q\left(T_{i}\right)\right) \mid N(t)=n\right] .
$$


Using the same notation and properties as those of the previous subsection, we have

$$
\begin{aligned}
\mathrm{E}\left[\left(\prod_{i=1}^{N(t)} q\left(T_{i}\right)\right) \mid N(t)=n\right] & =\mathrm{E}\left[\left(\prod_{i=1}^{N^{*}(s)} q\left(m^{-1}\left(T_{i}^{*}\right)\right)\right) \mid N^{*}(s)=n\right] \\
& =\mathrm{E}\left[\left(\prod_{i=1}^{n} q\left(m^{-1}\left(V_{(i)}\right)\right)\right)\right] \\
& =\left[\mathrm{E}\left(q\left(m^{-1}(s U)\right)\right)\right]^{n}
\end{aligned}
$$

and

$$
\mathrm{E}\left(q\left(m^{-1}(s U)\right)\right)=\frac{1}{m(t)} \int_{0}^{t} q(x) v(x) \mathrm{d} x
$$

Therefore,

$$
\mathrm{E}\left[\left(\prod_{i=1}^{N(t)} q\left(T_{i}\right)\right) \Psi(N(t)) \mid N(t)=n\right]=\Psi(n)\left(\frac{1}{m(t)} \int_{0}^{t} q(x) v(x) \mathrm{d} x\right)^{n} .
$$

Finally, we obtain a rather cumbersome relationship:

$$
\begin{aligned}
\mathrm{P}\left(T_{S}>t\right)= & \sum_{n=0}^{\infty} \Psi(n)\left(\frac{1}{m(t)} \int_{0}^{t} q(x) v(x) \mathrm{d} x\right)^{n} \frac{m(t)^{n}}{n !} \exp \{-m(t)\} \\
= & \sum_{n=0}^{\infty}\left(\sum_{j=n}^{\infty} \frac{(\eta(b-t))^{j}}{j !} \exp \{-\eta(b-t)\}\right)\left(\frac{1}{m(t)} \int_{0}^{t} q(x) v(x) \mathrm{d} x\right)^{n} \\
& \times \frac{m(t)^{n}}{n !} \exp \{-m(t)\}
\end{aligned}
$$

It can be easily shown that the survival function in (10) can be written in the following compact form:

$$
\mathrm{P}\left(T_{S}>t\right)=\exp \left\{-\int_{0}^{t} p(x) v(x) \mathrm{d} x\right\} \sum_{n=0}^{\infty} \mathrm{P}\left(Z_{1} \geq n\right) \mathrm{P}\left(Z_{2}=n\right),
$$

where $Z_{1}$ and $Z_{2}$ are two Poisson random variables with parameters $\eta(b-t)$ and $\int_{0}^{t} q(x) v(x) \mathrm{d} x$, respectively.

The following remark presents a qualitative analysis for two marginal cases of (11) for each fixed $t<b$.

Remark 4. (i) When $\eta=1 / \mu \rightarrow \infty$, which means that the mean of the increments $W_{i}$ tends to 0, (11) 'reduces' to the Brown-Proschan model, (2). Indeed, as $\eta \rightarrow \infty$,

$$
\sum_{n=0}^{\infty} \mathrm{P}\left(Z_{1} \geq n\right) \mathrm{P}\left(Z_{2}=n\right) \rightarrow \sum_{n=0}^{\infty} \mathrm{P}\left(Z_{2}=n\right)=1
$$

because $\mathrm{P}\left(Z_{1} \geq n\right) \rightarrow 1$ for all $n \geq 1$ and $\mathrm{P}\left(Z_{1} \geq 0\right)=1$. From 'physical considerations', it is also clear that as increments vanish, their impact on the model also vanishes, and, therefore it should reduce to the Brown-Proschan model. 
(ii) Now consider another marginal case when $\eta \rightarrow 0$, which means that the mean of the increments tends to $\infty$, and, therefore, the first shock will kill the system with probability tending to 1 as $\eta \rightarrow 0$. The infinite sum on the right-hand side of the following equation vanishes in this case:

$$
\begin{aligned}
\sum_{n=0}^{\infty} \mathrm{P}\left(Z_{1} \geq n\right) \mathrm{P}\left(Z_{2}=n\right) & =\mathrm{P}\left(Z_{1} \geq 0\right) \mathrm{P}\left(Z_{2}=0\right)+\sum_{n=1}^{\infty} \mathrm{P}\left(Z_{1} \geq n\right) \mathrm{P}\left(Z_{2}=n\right) \\
& \rightarrow \mathrm{P}\left(Z_{2}=0\right)
\end{aligned}
$$

as $\mathrm{P}\left(Z_{1} \geq 0\right)=1$ and $\mathrm{P}\left(Z_{1} \geq n\right) \rightarrow 0$ for all $n \geq 1$ when $\eta \rightarrow 0$. Therefore, finally,

$$
\begin{aligned}
\mathrm{P}\left(T_{S}>t\right) & \rightarrow \exp \left\{-\int_{0}^{t} p(x) v(x) \mathrm{d} x\right\} \exp \left\{-\int_{0}^{t} q(x) v(x) \mathrm{d} x\right\} \\
& =\exp \left\{-\int_{0}^{t} v(x) \mathrm{d} x\right\}
\end{aligned}
$$

which is a probability that no shocks have occurred in $[0, t]$. This is what we also expect from general considerations for $\eta \rightarrow 0$, as the system can survive for $t<b$ only without shocks.

\section{Concluding remarks}

In this paper, a new shock model that combines an extreme shock model with a specific cumulative shock model was proposed and analyzed. The considered setting can also be interpreted as a generalization of the well-known Brown-Proschan model that describes the corresponding repair process in terms of perfect and minimal repairs.

Although mathematical derivations are relatively cumbersome, the explicit self-explanatory relationships for the probability of nontermination of the nonhomogeneous Poisson process (and for the corresponding failure rate) have been derived for important special cases. Exponentially distributed and deterministic boundary cases were considered and some meaningful interpretations and remarks were discussed.

The considered approach can be generalized to the nonidentically distributed sequence of wear increments (e.g. forming a geometric process). Another possibility is to extend the perfect-minimal repair reasoning in Cha (2001) to the case when repair is worse than minimal. The assumption of an exponentially distributed boundary seems to be rather restrictive, but it allows for simple self-explanatory results. The case of a deterministic boundary considered in Subsection 3.2 is probably more realistic in this context.

\section{Acknowledgements}

The authors are very grateful to the anonymous referee for helpful comments and careful reading of this paper, which have considerably improved its presentation. The authors are also grateful to Miss Jeong Min Hyeon for editing the formulae in this paper.

The work of the first author was supported by a Korea Science and Engineering Foundation (KOSEF) grant funded by the Korea government (MOST) (grant number R01-2008-00010957-0). The work of the second author was supported by the National Research Foundation of South Africa (grant number FA2006040700002). 


\section{References}

Beichelt, F. E. AND Fischer, K. (1980). General failure model applied to preventive maintenance policies. IEEE Trans. Reliab. 29, 39-41.

Block, H. W., Borges, W. S. And Savits, T. H. (1985). Age-dependent minimal repair. J. Appl. Prob. 22, $370-386$.

Brown, M. and Proschan, F. (1983). Imperfect repair. J. Appl. Prob. 20, 851-859.

Cha, J. H. (2001). Burn-in procedures for a generalized model. J. Appl. Prob. 38, 542-553.

ÇInlaR, E. (1975). Introduction to Stochastic Processes. Prentice Hall, Englewood Cliffs, NJ.

Cox, D. R. And Isham, V. (1980). Point Processes. Chapman and Hall, New York.

Finkelstein, M. S. (1999). Wearing-out components in variable environment. Reliab. Eng. System Safety 66, $235-242$.

Finkelstein, M. S. (2007). On some ageing properties of general repair processes. J. Appl. Prob. 44, 506-513.

Finkelstein, M. S. (2008). Failure Rate Modelling for Risk and Reliability. Springer, London.

Gut, A. And Husler, J. (2005). Realistic variation of shock models. Statist. Prob. Lett. 74, 187-204.

Nachlas, J. A. (2005). Reliability Engineering. CRC Press, Boca Raton, FL.

Sumita, U. and Shanthikumar, J. G. (1985). A class of correlated cumulative shocks models. Adv. Appl. Prob. 17, 347-366. 